\title{
MULTI GRID CHAOTIC ATTRACTORS WITH DISCRETE JUMPS
}

\author{
Tomáš Gotthans ${ }^{* *}$ Z Zdeněk Hruboš ${ }^{*}$
}

\begin{abstract}
In this paper the discrete step functions are used in order to generate $\mathrm{m} \times \mathrm{n}$ scroll chaotic hypercube attractors. The design and realization of multi-scroll attractors depends on synthesizing the nonlinearity with an electrical circuit. The essence of the novel approach is in designing the transfer function with analog to digital converters connected directly without any microcomputer, instead of using standard comparator or hysteresis methods. Therefore there is no special need for synthesizing the nonlinearity towards $m \times n$ scroll chaotic attractors. The approach is verified with PSpice 16.0 circuit simulator and experimentally measured.
\end{abstract}

K e y w or d s: multi grid scrolls, chaos, dynamical systems, integrator synthesis, digital converters

\section{INTRODUCTION}

Over past three decades, generating multi-scroll chaotic attractors became an aim of many researchers [2-5, 10]. Many techniques involving different approaches (usually using comparators or hysteresis) have been published [1, 11]. Chaos control and generation has a dramatic increase of interest since many real world applications and observations in engineering or other fields have been presented. For example in fields such as bio-medical engineering, digital data encryption, power systems protection, re-configurable hardware, and so on. But yet there is no simple rule for quantifying chaos origin. Generating chaotic attractors may help to understand better dynamics of real world systems. In the article we would like to study third order nonlinear system, where such behavior is very rare [6]. We are presenting a generalized method for generating $2 \mathrm{D} \mathrm{m} \mathrm{x} \mathrm{n} \mathrm{grid} \mathrm{scroll,} \mathrm{where} \mathrm{a} \mathrm{special} \mathrm{case}$ of solution is set of $1 \mathrm{D}$ grid scrolls $[8,12]$. The chosen $2 \mathrm{D}$ $\mathrm{m} \times \mathrm{n}$ scroll attractor can be in fact considered as particular case of Chuas attractor [7]. Of course similar approach can be utilized for 3D grid scrolls by adding another nonlinear functional block. Our solution involves only analog to digital converters $(\mathrm{AD})$ and digital to analog converters (DA) for implementation of the non-linear function. It comes to this, that there is no need for any microcontroller.

The model describing chaotic $2 \mathrm{D} \mathrm{m} \times \mathrm{n}$ scroll generation is described by three first-order differential equations.

$$
\dot{\mathbf{x}}=\mathbf{A} \boldsymbol{x}+\mathbf{B} \varphi\left(\mathbf{C}_{\boldsymbol{x}}\right) .
$$

Matrices A, B, C and function $\varphi($.$) are$

$$
\begin{gathered}
\mathbf{A}=\left(\begin{array}{ccc}
0 & 1 & 0 \\
0 & 0 & 1 \\
-a & -b & -c
\end{array}\right), \mathbf{B}=\left(\begin{array}{ccc}
0 & -1 & 0 \\
0 & 0 & -1 \\
a & b & c
\end{array}\right) \\
\mathbf{C}=\left(\begin{array}{ccc}
1 & 0 & 0 \\
0 & 1 & 0 \\
0 & 0 & 1
\end{array}\right), \varphi=\left(\begin{array}{c}
f(x) \\
f(y) \\
0
\end{array}\right)
\end{gathered}
$$

For numerical integration the fourth-order numerical Runge-Kutta method with variable step is used. $\dot{x}$ represents first order derivative. Function $f(\cdot)$ denotes a nonlinear step function. Parameters $a, b$ and $c$ are constants. For synthesis of the nonlinear step function, connecting the ADC directly with the DAC generate step transfer function. Defining step

$$
\Delta=\frac{\text { Dynamical range }(\mathrm{V})}{\text { Number of bits }} \text {. }
$$

Then output value with steps is

$$
\begin{gathered}
\operatorname{out}(x)= \begin{cases}l \Delta+\frac{\Delta}{2} & \text { if } x>0, \\
l \Delta-\frac{\Delta}{2} & \text { if } x<0,\end{cases} \\
l=\frac{x}{\Delta} \wedge l \in \mathbb{N}
\end{gathered}
$$

and $\mathbb{N}$ stands for set of natural numbers. Then model representing $\mathrm{ADC}$ connected directly to DAC, the step function with saturation can be written as

$$
f(x)= \begin{cases}\operatorname{out}(x) & \text { if }|x|<\Psi \\ -\Psi+\frac{\Delta}{2} & \text { if } x \leq-\Psi \\ \Psi-\frac{\Delta}{2} & \text { if } x \geq \Psi\end{cases}
$$

where $\Psi=\frac{\text { Dynamical range }(\mathrm{V})}{2}$.

\footnotetext{
* Department of Radio Electronics, Brno University of Technology, Purkynova 118, 61200 Brno, Czech Republic, ${ }^{* *}$ ESIEE, Universite Paris Est Marne la Vallee, boulevard Blaise Pascal 2, 93162 Noisy le Grand CEDEX, France, tomas.gotthans@phd.feec.vutbr.cz, xhrubo00@stud.feec.vutbr.cz
} 


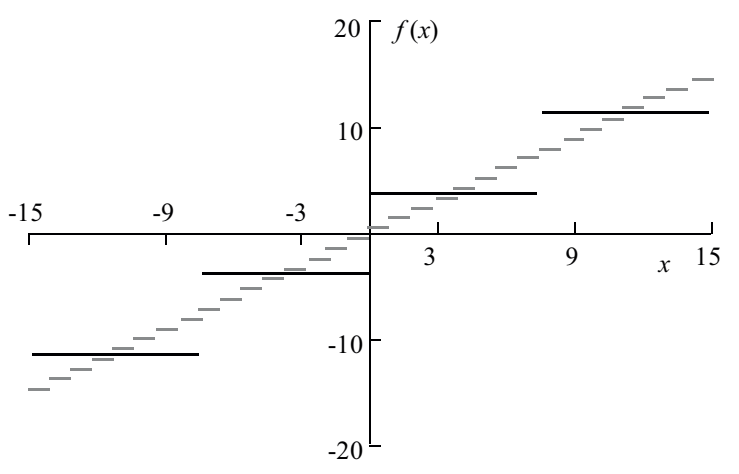

Fig. 1. The model of step function $f(x)$ for $2 \mathrm{~b}$ (black) and for $5 \mathrm{~b}$ (gray)

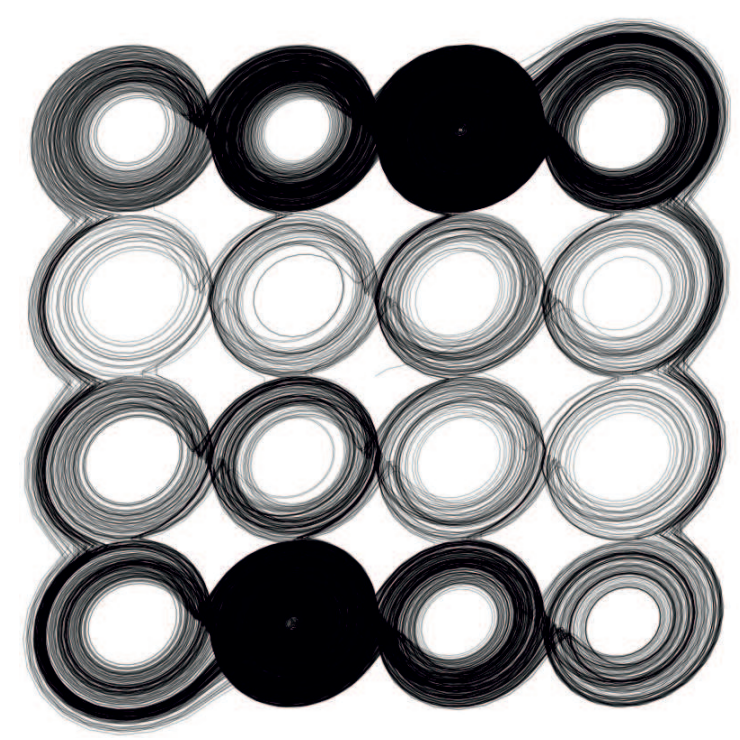

Fig. 2. The numerically integrated system (1), the Monge's projections $V(x)$ vs $V(y)$

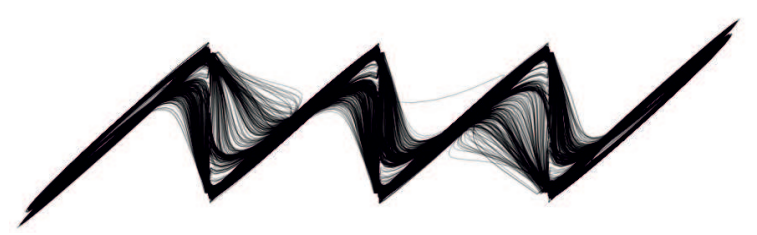

Fig. 3. The numerically integrated system (1), the Monge's projections $V(y)$ vs $V(z)$

A system (1) with function (7) and with constants set to $a=b=c=0.8$ can bee seen in Fig. 2 and Fig. 3 . Where the both functions (7) consists of 4 levels, ie is equal to utilizing 2 bit $\mathrm{AD} / \mathrm{DA}$ converters.

\section{CIRCUIT}

Synthesis of the electronic circuits is the easiest way how to accurately model the nonlinear dynamical systems. There exist several ways how to practically realize chaotic oscillators. Most of these techniques are straightforward and have been already published. To synthesize circuit from differential equations system (1), integrator synthesis was chosen. After thinking about how to reduce the complexity of the nonlinear network a very simple circuitry has been revealed. Only few basic building blocks are necessary: inverting integrators, summing amplifier, $\mathrm{AD}$ and DA converters and voltage sources. Electronic circuit system consists of three integrator circuits (using operational amplifier AD713), which integrate (1). Values of passive parts are estimated directly from the equations. The synthesized schematics is in Fig. 4. In order to ensure NyquistShannon sampling criterion for the converters, frequency re-normalization is an easy and straightforward process covering identical change of all integration constants simultaneously.

To create step transfer functions $f(x)$ and $f(y)$, the data converters are used. The schematics in Fig. 5 shows the data converters connected directly to produce step transfer function. In order to process positive and negative voltages, the circuit is divided in the two branches. Voltage sources are used as references for the converters. The circuitry realization was evaluated using OrCAD PSpice. The overall simulation time is set to $100 \mathrm{~ms}$. The simulated output of Monge's projections is in the in Figs. 6-8. The values of passive resistors are $R_{1}=$ $R_{6}=R_{7}=125 \mathrm{k} \Omega, R_{5}=R_{8}=R_{9}=R_{13}=R_{14} 100 \mathrm{k} \Omega$, $R_{2}=R_{3}=R_{4}=1 \mathrm{k} \Omega, R_{10}=R_{11}=118 \mathrm{k} \Omega, R_{12}=$ $1 \mathrm{k} \Omega, R_{\text {Out }}=1 \Omega$ and values of the capacitors are $C_{1}=C_{2}=C_{3}=100 \mathrm{nF}$.

Towards to produce various number (less) of levels for the step function, one possibility is to use only certain number of bits between converters. Another possibility is to invoke Boolean logical functions between converters (can be implemented eg in FPGA).

\section{EXPERIMENTAL RESULTS}

It should be pointed out that hardware implementation of $2-\mathrm{D} m \times \mathrm{n}$ scroll chaotic attractors is technically very difficult $[1,9]$, despite there is no theoretical limitation in the mathematical model for generating the large numbers of the multidimensional scrolls. The above circuit design method provides a theoretical principle for hardware implementation of such chaotic attractors with multidirectional orientations and a satisfactory number of scrolls. The measurements presented in Figs 9-14 were done using HP 54645D oscilloscope.

\section{3D GRID SCROLLS}

By simple modification of the matrix $\mathbf{B}$ and the matrix function $\varphi(\cdot)$

$$
\mathbf{B}=\left(\begin{array}{ccc}
0 & -1 & 0 \\
0 & 0 & -1 \\
d & b & c
\end{array}\right), \varphi=\left(\begin{array}{l}
f(x) \\
f(y) \\
f(z)
\end{array}\right),
$$

one can obtain 3D $(k, l, m)$ grid scrolls by setting $a=$ $b=c=0.8$ and $d=0.77$. The constants $k, l, m$ stand for the number of levels of the nonlinearity (7). 


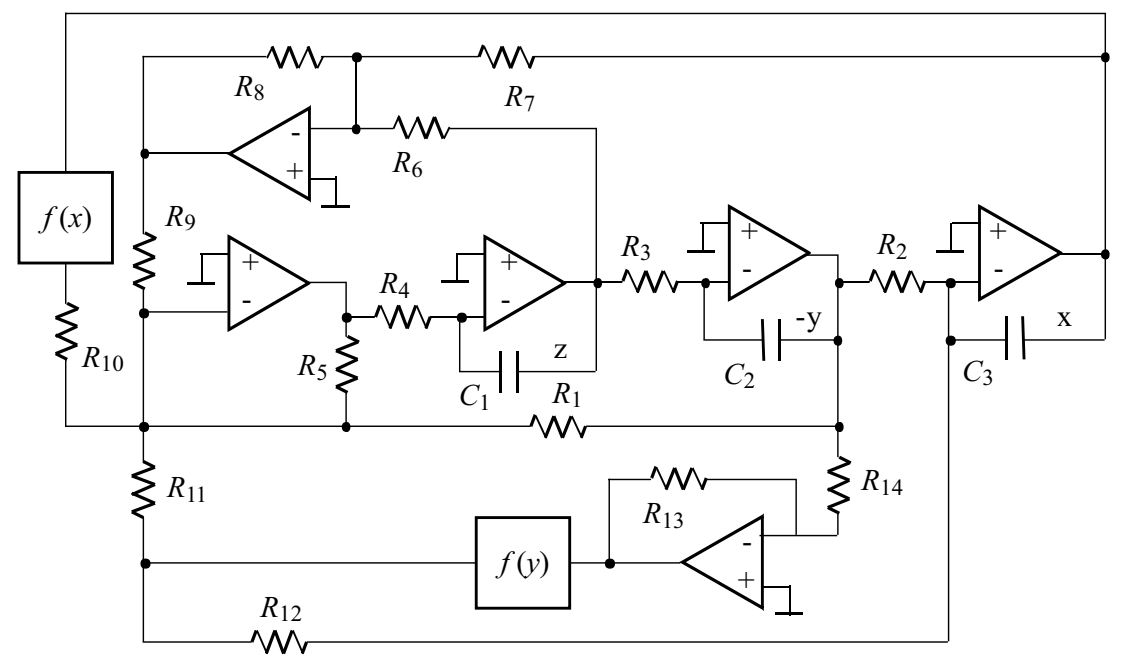

Fig. 4. The block schematics of realization of (1)

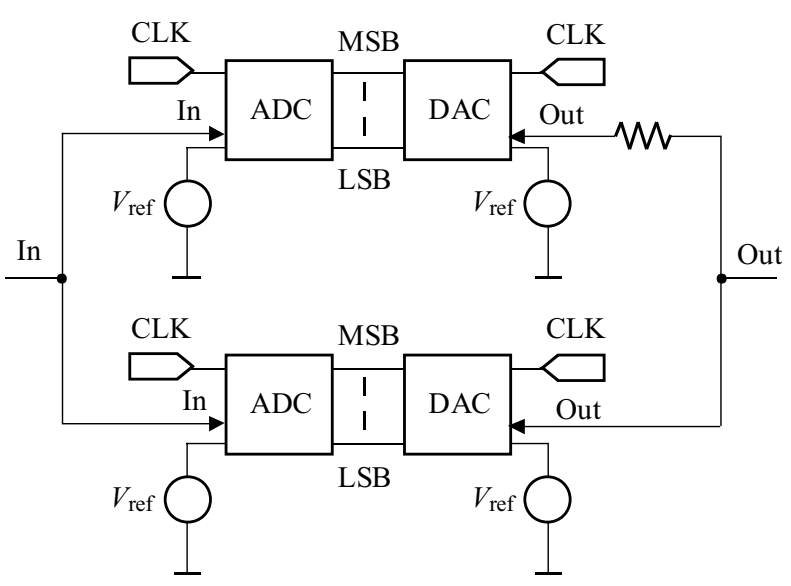

Fig. 5. The block schematics of realization of function $f(x)$ using data converters

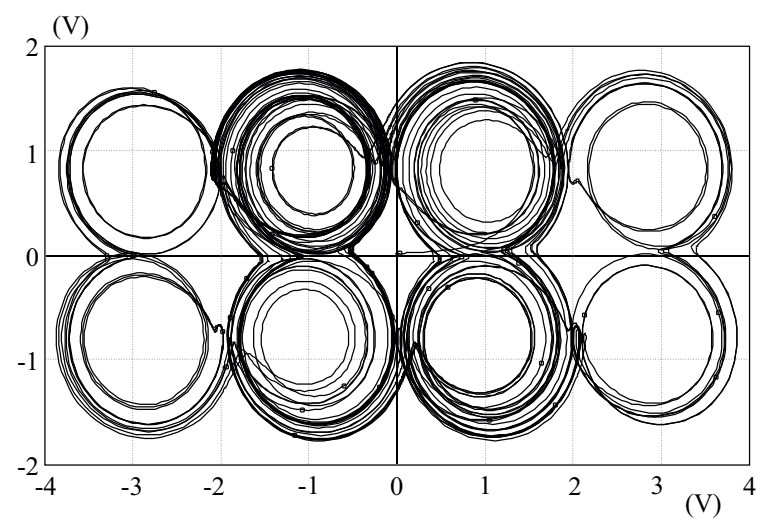

Fig. 7. The simulations from PSpice program, $V(x)$ vs $V(y)$ projections

\section{CONCLUSION}

Generating chaotic behavior in third-order autonomous systems is quite delicate process. The whole system is extremely sensitive as for the initial conditions as for the realization. It is known that avoiding fractional integra-

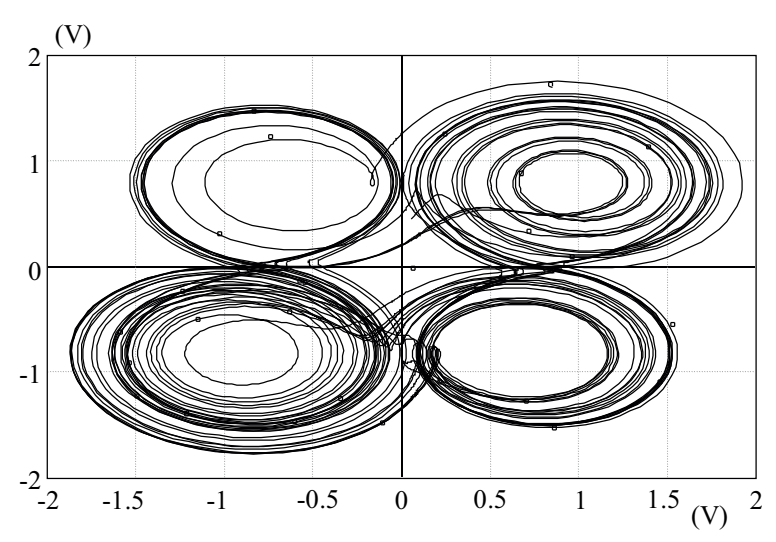

Fig. 6. The simulations from PSpice program, $V(x)$ vs $V(y)$ projections

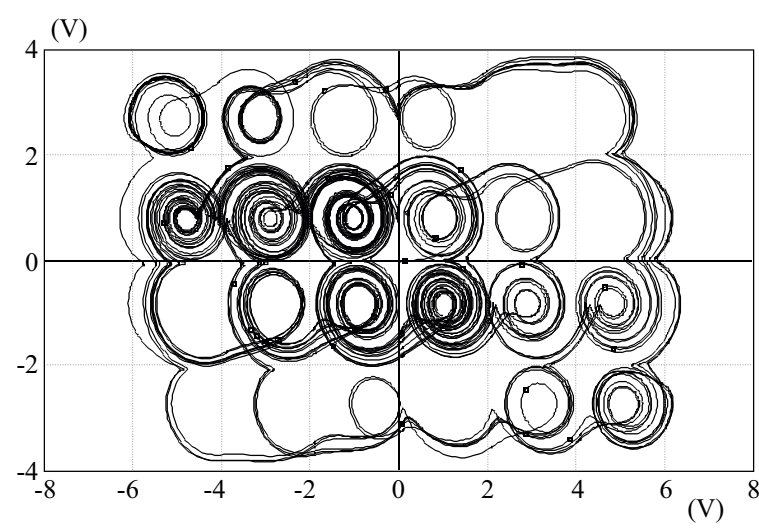

Fig. 8. The simulations from PSpice program, $V(x)$ vs $V(y)$ projections

tors the third order of autonomous dynamical systems is the minimum order to produce chaos. To obtain chaotic behavior, the whole system has to be perfectly balanced. With the growing order of the system, the presence of chaotic behavior is more probable. In this paper the well known 2-D $m \times n$ scroll system was chosen and was re- 


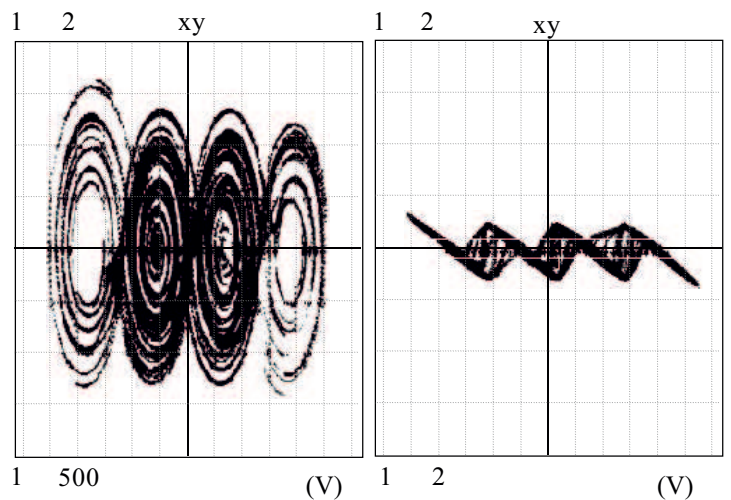

Fig. 9. Special setup where step function $f(y)$ vanishes: projections $V(x)$ vs $V(-y)$ (left), $V(-y)$ vs $V(z)$ (right)

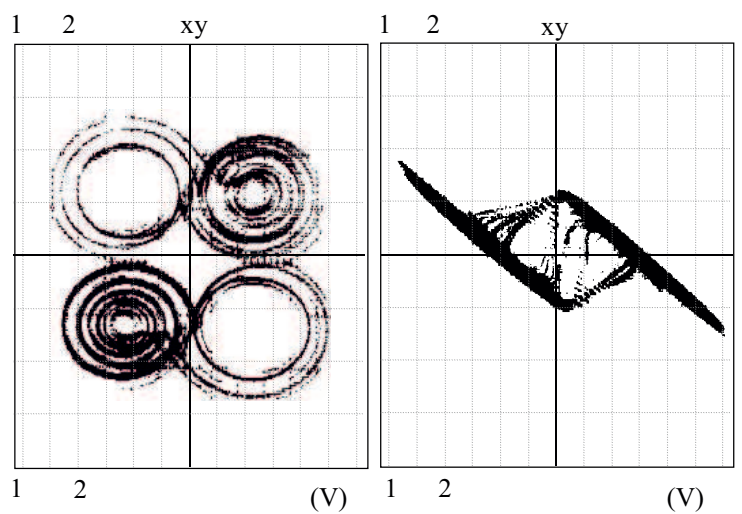

Fig. 11. Measured system, $2 \times 2$ scroll: projections $V(x)$ vs $V(-y)$ (left), $V(-y)$ vs $V(z)$ (right)

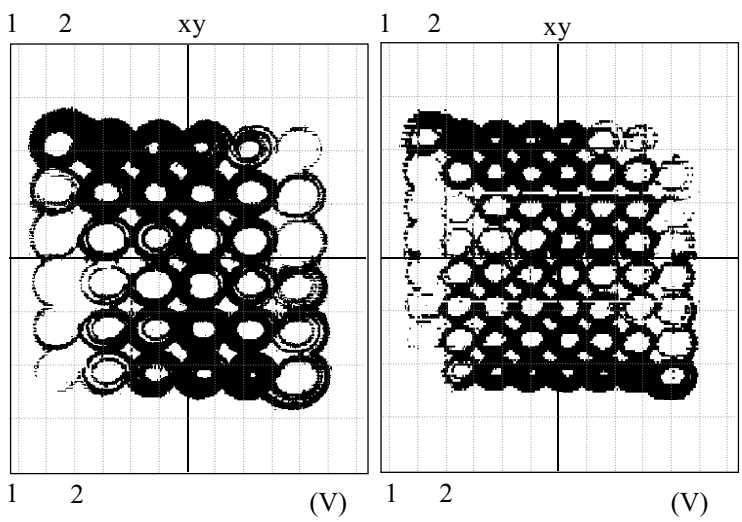

Fig. 13. Measured system, $6 \times 6$ scroll: projections $V(x)$ vs $V(-y)$ (left), $8 \times 8$ scroll, projections $V(x)$ vs $V(-y)$ (right)

alized utilizing novel approach using the data converters as non-linear functions. First the models were derived to simulate the data converters connected directly (ADCDAC). Than the connection was reduced to produce less scrolls. To verify the chaotic behavior of proposed conception, the circuit simulator PSpice was used. Then the circuit was build and measured. The measured results are rather matching the theoretical expectations.

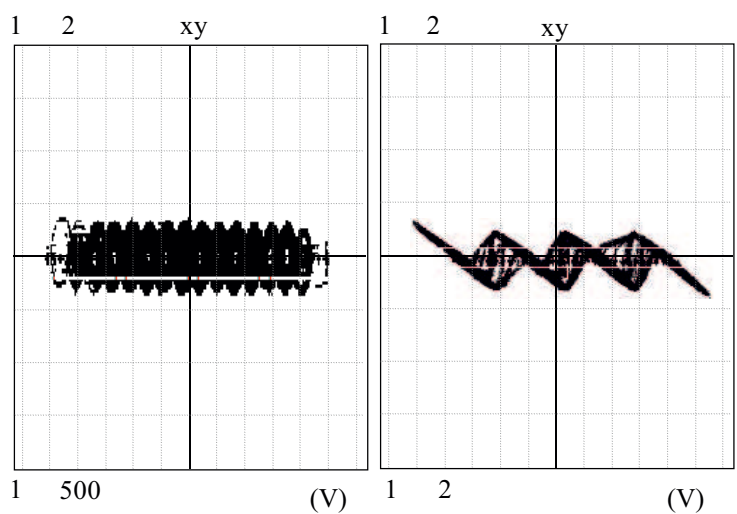

Fig. 10. 1D 16 scroll, step function $f(y)$ vanishes: projections $V(x)$ vs $V(-y)$ (left), $V(-y)$ vs $V(z)$ (right)

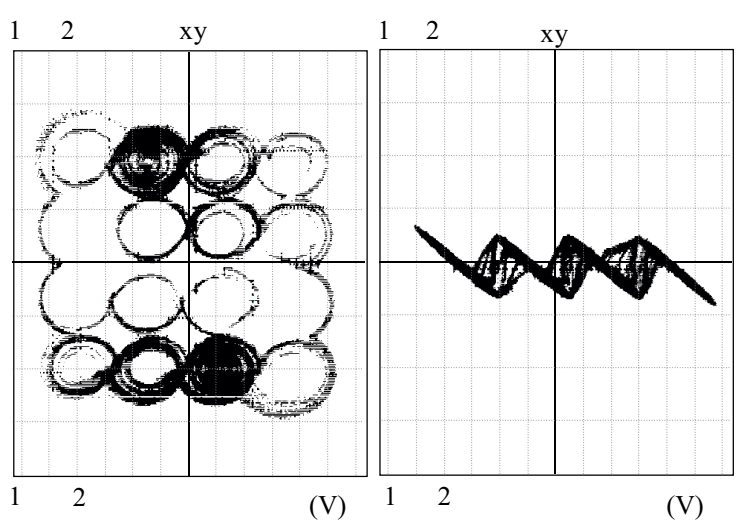

Fig. 12. Measured system, $4 \times 4$ scroll: projections $V(x)$ vs $V(-y)$ (left), $V(-y)$ vs $V(z)$ (right)

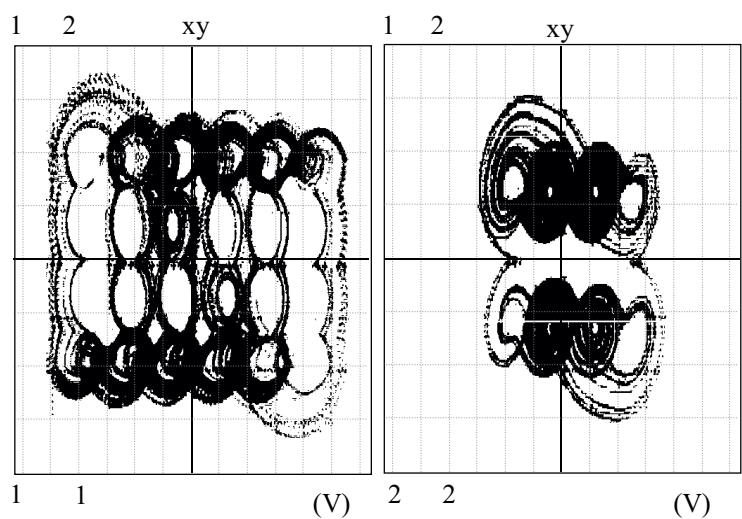

Fig. 14. Measured system - perturbation of parrameters, $6 \times 4$ scroll (left) and $4 \times 2$ scroll (right): projections $V(x)$ vs $V(-y)$

\section{Acknowledgements}

Research described in the paper was supported by the Czech Ministry of Education under research program MSM 0021630513. This work has also received funding partially from the operational program WICOMT denoted as CZ.1.07/2.3.00/20.0007. Measurements were performed in laboratories supported by the SIX project; the registration number CZ.1.05/2.1.00/03.0072, the op- 


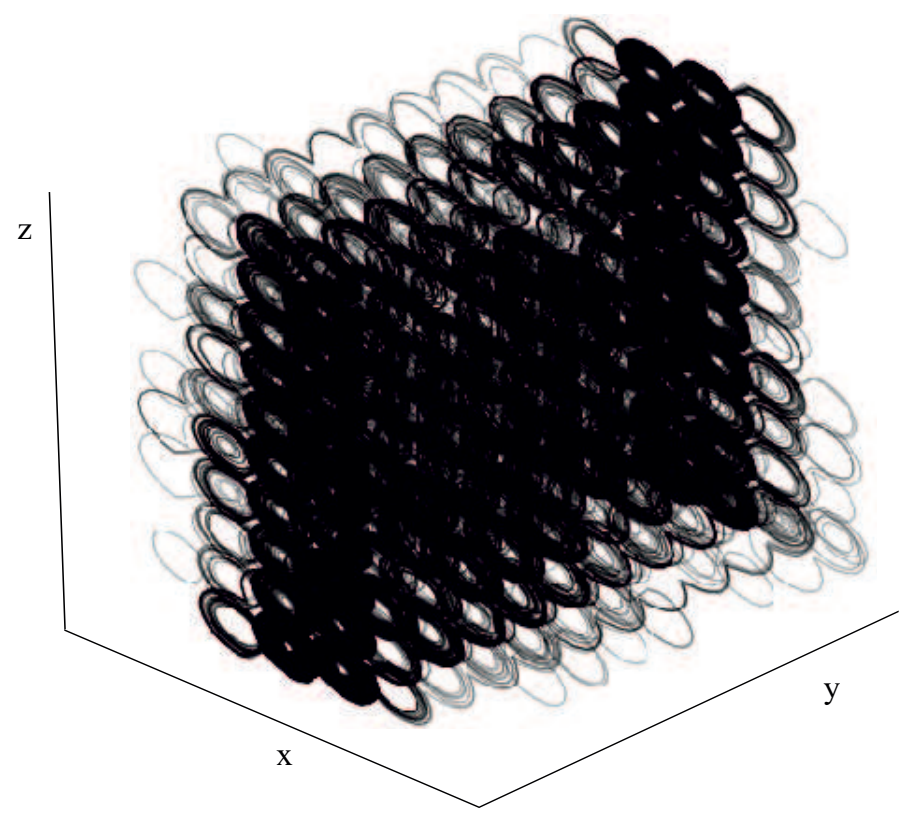

Fig. 15. Numerically simulated $3 \mathrm{D}(10,10,10)$ grid scolls

erational program Research and Development for Innovation.

\section{REFERENCES}

[1] LU, J.-CHEN, G. : Generating Multiscroll Chaotic Attractors: Theories, Methods and Applications, International Journal of Bifurcation and Chaos 16 No. 4 (2006), 775-858.

[2] PetrzelA, J.-PROKOPEC, J.: Some Practical Experiments with Simple Driven Dynamical Systems, Radioelektronika, 19th International Conference, 2009.

[3] Petrzela, J.-Hrubos, Z.-GotThans, T.: Modeling Deterministic Chaos using Electronic Circuits, Radioengineering 20 No. 2 (2011), 438-444.

[4] SPROTT, J. C.: Elegant Chaos: Algebraically Simple Chaotic Flows, World Scientific, Singapore, 2010.

[5] Petrzela, J.-HRubos, Z.-GOTthans, T.: Chaotic Oscillators with Single Polynomial Nonlinearity and Digital Sampled Dynamics, Przeglad Electrotechniczny 3 No. 1 (2011), 161-163.

[6] ELHADJ, Z.-SPROTT, JC.: Some Open Problems in Chaos Theory and Dynamics, International Journal of Open Problems in Computer Science and Mathematics 4 (2011), 1-10.

[7] SMALL, M.: Applied Nonlinear Time Series Analysis: Applications in Physics, Physiology and Finance, World Scientific Publishing Company, 2005.

[8] TREJO-GUERRA, R.-TLELO-CUAUTLE, E.-SANCHEZLOPEZ, C.-MUNOZ-PACHECOJ. M.-CRUZ-HERNANDEZ, C. : Realization of Multiscroll Chaotic Attractors by using
Current-Feedback Operational Amplifers, Revista Mexicana de Fisica 56 (2010), 268-274.

[9] LU, J.-HAN, F.-YU, X.-CHEN, G.: Generating 3-D Multi-Scroll Chaotic Attractors: A Hysteresis Series Switching Method, Automatica (2004), 1677-1687.

[10] AHMAD, W. M.: Generation and Control of Multi-Scroll Chaotic Attractors in Fractional Order Systems, Chaos, Solution \& Fractals 25 (2005), 727-735.

[11] OZOGUS, S.-ELWAKILA. S.-SALAMA, K. N.: n-Scroll Chaos Generator using Nonlinear Transconductor, Elecron Lett. 38 (2002), 685-686.

[12] SPANY, V.-GALAJDA, P.-GUZAN, M.-PIVKA, L.-OLEJAR, M.: Chua's Singularities: Great Miracle in Circuit Theory, I. J. Bifurcation and Chaos (2010), 2993-3006.

Received 31 July 2012

Tomáš Gotthans was born in Brno, Czech Republic, in 1985. He received the MSc degree at the Brno University of Technology in 2010. Now he is a PhD student at the Department of Radio Electronics, Brno University of Technology and ESIEE Paris. His research interests include nonlinear dynamics, linearization of power amplifiers and circuit synthesis.

Zdeněk Hruboš was born in Uherské Hradiště, Czech Republic, in 1984. He received his MSc degree in 2009 from the University of Technology, Brno. He is currently pursuing his $\mathrm{PhD}$ study at the same university. His research is aimed in the computer analysis and synthesis of electronics circuits and nonlinear systems. 